\title{
Intravenous Patient-Controlled Remifentanil Versus Paracetamol in Post-Operative Pain Management in Patients Undergoing Coronary Artery Bypass Graft Surgery
}

\author{
Alireza Jahangiri Fard ${ }^{1}$; Touraj Babaee ${ }^{2}$; Seyed Mostafa Alavi ${ }^{2}$; Ali Akbar Nasiri ${ }^{3}$; Seyed \\ Mohamad Mehran Ghoreishi ${ }^{2}$; Noor Mohammad Noori ${ }^{2}$; Maziar Mahjoubifard ${ }^{4, *}$ \\ ${ }_{2}^{1}$ Masih Daneshvari Institute of Tuberculosis and Lung Disease, Shahid Beheshti University of Medical Sciences, Tehran, Iran \\ ${ }_{3}^{2}$ Rajaie Cardiovascular Medical and Research Center, Iran University of Medical Sciences, Tehran, Iran \\ ${ }^{3}$ Department of Anesthesiology, Zabol University of Medical Sciences, Zabol, Iran \\ ${ }^{4}$ Research Center of Children and Adolescent Health, Ali-Ebn E-Abitaleb Hospital, Zahedan University of Medical Sciences, Zahedan, Iran \\ ${ }^{*}$ Corresponding author: Maziar Mahjoubifard, Research Center for Children and Adolescent Health, Ali-Ebn E-Abitaleb Hospital, Zahedan University of Medical Sciences, Zahedan, \\ Iran. Tel: +98-5412412780, Fax:+98-5413425563, E-mail: m_mahgoobifard@yahoo.com \\ ; Revised: June 5, 2014; Accepted: June 11, 2014
}

\begin{abstract}
Background: Pain management after cardiac surgery has been based on parenteral long-acting opioids such as morphine. The other alternatives are paracetamol and remifentanil.

Objectives: In this prospective, double-blind, randomized study, we compared the efficacy of intravenous patient-controlled analgesia (IV-PCA) paracetamol and remifentanil for post cardiac surgery pain relief.

Materials and Methods: One-hundred patients scheduled for elective coronary artery bypass grafting from May to October 2011, were randomized into two groups after the surgery. For the first group (group R, $\mathrm{n}=50$, with mean age of $58.16 \pm 11.80$ ), the IV-PCA protocol was remifentanil infusion $100 \mu \mathrm{g} / \mathrm{h}$; bolus of $25 \mu \mathrm{g}$ and lockout time of 15 minutes. In the second group (group P, $\mathrm{n}=50$, with mean age of 53.8 \pm 15.08 ), patients received paracetamol $15 \mathrm{mg} / \mathrm{kg}$ as a bolus at the end of surgery and then IV-PCA protocol was $100 \mu \mathrm{g} / \mathrm{h}$, bolus of $25 \mu \mathrm{g}$; and lockout time of 15 minutes. Pain was assessed with visual analog scale score (VAS) in the first 24 hours after surgery for seven times.

Results: The trend of pain scores did not have any significantly difference between group R and group P except for hour 8 and hour 18 after surgery that VAS was significantly lower in group $\mathrm{P}$ than group $\mathrm{R}(\mathrm{P}=0.031, \mathrm{P}=0.023$, respectively). Respiratory rate (RR) was also statistically lower in group R comparing to group P in all seven evaluating times. The groups were similar in terms of hemodynamic, ABG results (except for $\mathrm{PaO}_{2}$, which was significantly lower in group $\mathrm{R}$ than group P at 6 evaluating times), intubation time, renal function tests, and incidences of atelectasis, myocardial infarction or adverse effects.

Conclusions: Both PCA techniques provided effective pain scores ( $<3)$ after cardiac surgery; but generally, PCA-paracetamol infusion has a better analgesic effect.
\end{abstract}

Keywords:Remifentanil; Patient-Controlled Analgesia; Cardiac Surgery

\section{Background}

The pain after cardiac surgeries is due to different reasons. The most important reasons include sternotomy, different drains insertion, and saphenous vein harvesting (1). Poor pain management can lead to important post-operative complications such as myocardial ischemia following sympathetic nervous system stimulation, pulmonary events, atelectasis and pneumonia, vein thrombosis - because of the post-operative decreased motility of the patients and prolonged intensive care unit admission time. Therefore, pain management plays an important role in post-operative complications (2). Nowadays, in order to provide post-operative pain relief, intravenous long-acting opioids such as morphine are the most frequently used analgesics, which are performed with different techniques including manually titrated continuous infusion or intra venous patient-controlled analgesia (IV-PCA). However, some important side effects of these analgesics are drowsiness, prolonged and excessive sedation, respiratory depression, nausea and vomiting (2). Furthermore, non-steroidal anti-inflammatory drugs (NSAIDs) are used as alternative therapy in postcardiac surgeries' pain management. These analgesics are related to different complications (increased post-operative bleeding, renal dysfunction) (1). Nowadays, the PCA method results into better postoperative pain management and less analgesics requirement (1). Remifentanil is an analgesic, which is used with this method (3). This selective $\mu$-opoid agonist has a fast onset and an ultra-short duration of action with peak of effect in the first $15 \mathrm{~s}$ and its duration of its effect is in 3-4 minutes. Remifentanil is metabolized by non-specified esterase and is not dependent on liver or renal metabolism. In order to control the

Copyright (C) 2014, Iranian Society of Regional Anesthesia and Pain Medicine (ISRAPM); Published by Kowsar. This is an open-access article distributed under the terms of the Creative Commons Attribution-NonCommercial 4.0 International License (http://creativecommons.org/licenses/by-nc/4.0/) which permits copy and redistribute the material just in noncommercial usages, provided the original work is properly cited. 
possible incidence of overdose toxicity of this analgesic, the dosage can be decreased rapidly and the situation of the patient returns to normal level in a short time, since remifentanil is not concentrated in organs $(4,5)$. However, the safety of remifentanil in awake and spontaneously breathing patients in post-operative period is still a controversy and it can cause respiratory depression in some cases (3). In addition, the cessation of this opioid can result into hyperanalgesia in first few minutes because of withdrawal of the analgesic effect $(3,6-8)$. Paracetamol is another safe analgesic, which is used to control the pain after the surgeries in patients. This analgesic can also be used by PCA. The onset of analgesia is 5-10 minutes and its half-life is 1-4 hours. The recent findings suggest that it is highly selective for COX-2 (9) and is primarily metabolized in liver into non-toxic products. The adverse effects caused by opioids or NSAIDs are not reported in this analgesic (3).

\section{Objectives}

The aim of this study was to evaluate and compare the effects of IV-PCA of remifentanil and paracetamol and to determine patient satisfaction with these treatments.

\section{Materials and Methods}

After approval of the study protocol by the institutional review board, and obtaining written informed consent, 100 patients (male: 51, female: 49 with mean age of $56 \pm$ 13.65) who had American Society of Anesthesiologists (ASA) physical status classification of I to III were scheduled to undergo elective primary CABG at Shahid Rajaei Heart Hospital. These patienrs were randomly divided into two groups prospectively from May to October 2011. Randomization was performed with the closed envelope technique. All patients were blinded to their treatment group. The inclusion criteria were as follows: pre-operative ejection fraction (EF) $>30 \%$, ASA class $<$ III, or not having concomitant valve surgery, emergency procedures, pre-operative liver function tests two times higher than the reference range, chronic renal insufficiency (a serum creatinin level of $>2 \mathrm{mg} / \mathrm{dL}$ ), no use of intra-aortic balloon pump (IABP) during the surgery, opium addiction, diabetes mellitus, intra-operative sternal or rib fracture, pre-operative neurological symptoms or complications and not having impaired level of consciousness. The patients received the same premedication (morphine 0.1 $\mathrm{mg} / \mathrm{kg} / \mathrm{IM}$ and lorazepam $1 \mathrm{mg} / \mathrm{po}$ ). After ECG monitoring and arterial line insertion, anesthesia was induced for all patients with remifentanil $(1 \mu / \mathrm{kg})$, midazolam $(0.1 \mathrm{mg} /$ $\mathrm{kg})$ and then atracurium $(0.15 \mathrm{mg} / \mathrm{kg})$. Afterwards, intratracheal intubation was performed; central vein (CV) line and Foley catheter were also inserted for all the patients. The anesthesia was maintained with a remifentanil infusion (0.05 -0.1 $\mu \mathrm{g} / \mathrm{kg} /$ minute), midazolam (0.02-0.05 $\mu \mathrm{g} /$ $\mathrm{kg} /$ minute) and then atracurium and infusion of propofol if necessary. The surgeries were done uniformly by only one surgical unit and according to standard procedures (midline sternotomy and moderate hypothermia). Before and during the cardiopulmonary bypass (CPB), activated clotting time (ACT) was maintained over 480 seconds. The patients were weaned from $\mathrm{CPB}$ under standard protocol and epinephrine was used as an alternative inotrope in case of necessity. After the operation, the patients were randomly divided in two equal groups. The drugs were prepared by an anesthesiologist and before transferring the group A patients (male: 22 , female: 28 , with mean age of $58.16 \pm 11.80)$ to the intensive care unit (ICU), remifentanil $(25 \mu \mathrm{g} / \mathrm{cc})$ was administered with IV-PCA infusion pump ( 4 cc/hour - Royal Fornia M.E, China-US). In contrast the group B patients (male: 29 , female: 21 with mean age of $53.8 \pm 15.08)$ received a bolus dose of paracetamol (15 $\mathrm{mg} / \mathrm{kg} / \mathrm{IV})$ and then the infusion of paracetamol $(25 \mu \mathrm{g} /$ cc, 4 cc/hour) was administered. Afterwards, the patients were transferred to the ICU, where they were sedated by bolus dose of midazolam and weaned from mechanical ventilation after achieving the standard criteria. During the first 24 hours after the surgery, all patients received continuous IV-PCA opioid infusions (group A: remifentanil, group B: paracetamol). For all the patients who suffered from pain, the analgesics were also administered as a bolus dose of analgesics (1 cc) and a locked out time of 15 minutes. The intensity of pain, hemodynamic criteria (BP: blood pressure, HR: heart rate), arterial blood gas (ABG) results and serum lactate level were assessed for all the patients two hours after transferring the cases to the ICU with 2-6 hours' time intervals (two, four, six, 12,18 and 24 hours after transferring the cases to the ICU). The intensity of pain was assessed by visual analog scale. In this method the patients were asked to determine the score of their pain from one to 10 and for whom that could not participate in this method, one registered nurse determine the pain score based on the facial expression of the patients and their related figures in an illustrative score system. The other evaluating criteria in 24 hours after the surgery were analgesics' adverse effects (itching, nausea and vomiting), respiratory depression (RR: respiratory rate $<8$ ), duration of intubation, the incidence of postoperative liver and/or renal dysfunction (liver dysfunction: results of the liver function test 2 times more than the reference range, renal dysfunction: a serum creatinin level of $>2 \mathrm{mg} / \mathrm{dL}$ ), the incidence of post-operative myocardial infarction (MI) and atelectasis, which was visible in chest X-rays (CXR).

\subsection{Statistical Analysis}

Statistical analyses were performed with SPSS software version 15 . Clinical data are expressed as mean values \pm standard deviation. Differences were analyzed with independent student's T-test for the values of a scaling term and Pearson's chi square test for nominal values. MannWhitney-U test was used for comparing the values without normal distribution between two groups. 


\section{Results}

All 100 patients (50 in group R: Remifentanil and 50 in group P: Paracetamol) were accepted as study subjects. Preoperative characteristics and risk assessment and intra-operative data were similar in both groups (Table 1). The duration of mechanical ventilation was similar in both groups (group R: $8.63 \pm 3.3 \mathrm{~h}$ vs. $9.24 \pm 2.8 \mathrm{~h}$ in group $\mathrm{P})(\mathrm{P}=0.468)$. The mean dose of remifentanil administration was $2.35 \pm 0.25 \mathrm{mg}$ and the mean dose of paracetamol administration was $3.48 \pm 0.67 \mathrm{~g}$ in 24 hours of the study period. There were no significant differences in mean values of hemodynamic variables (Blood pressure: BP, Heart rate: HR) between groups at different seven evaluating times (Table 2). Table 2 also shows that respiratory rate (RR) was significantly lower in group $\mathrm{R}$ comparing to group $\mathrm{P}$ at all seven evaluating times $(\mathrm{P}<0.005)$. The mean values of visual analog scale score (VAS) were significantly lower in group comparing to group R at 8 and 18 hours after the surgery (1.08 \pm 1.92 in group R vs 0.28 \pm 0.60 in group $P$ at 8 hours and $1.04 \pm 1.80$ in group $R$ vs $0.30 \pm 0.78$ in group $\mathrm{P}$ at 18 hours, $\mathrm{P}<0.005)$.No significant differences were observed between the 2 groups with regard to VAS scores at other evaluating times (Table 3). The ABG analysis results showed that $\mathrm{PaO} 2$ was significantly lower in group $\mathrm{R}$ than group $\mathrm{P}(\mathrm{P}<0.005)$ at 6 different evaluating times (hours 2, 4, 6, 8, 12, 18); however, there was no significant difference regarding this variable between groups at hour 24 (Table 4). Also, there were no significant differences between groups with regard to $\left(\mathrm{PH}, \mathrm{PaCO}_{2}\right)$ during 24 hours study period but the mean values of serum lactate level was statistically higher in group R at 2, 4, 6, 18, 24 hours after the surgery $(\mathrm{P}<0.005)$. The number of complications in the postoperative periods did not differ statistically between the groups during 24 hours study period (Table 5). Post-operative first day chest radiograph revealed atelectasis in 15 (30\%) in group R patients vs 13 (26\%) in group P patients, but no intervention other than routine chest physiotherapy was needed in these individuals. Also postoperative first-day myocardial ischemia was occurred in five patients of each group. The intervention for these patients was based on the same protocol without analgesics administration.

Table 1. Characteristics of the Patients, Risk Assessment and Intraoperative Data ${ }^{\text {a,b }}$

\begin{tabular}{lccc}
\hline & $\begin{array}{c}\text { Group R(n } \\
=\mathbf{5 0})\end{array}$ & $\begin{array}{c}\text { Group P (n } \\
\mathbf{= 5 0})\end{array}$ & P Value \\
\hline Age, $\mathbf{y}$ & $58.16 \pm 11.80$ & $53.8 \pm 15.08$ & 0.111 \\
Height, cm & $165.24 \pm 10.08$ & $161.78 \pm 7.91$ & 0.061 \\
Weight, kg & $70.06 \pm 13.08$ & $70.06 \pm 13.38$ & 1.000 \\
\hline Sex, male, \% & $22(44)$ & $28(56)$ & 0.161 \\
Hypertension, \% & $28(56)$ & $30(60)$ & 0.263 \\
Ejection fraction & $47.40 \pm 7.70$ & $47.55 \pm 9.07$ & 0.906 \\
Bypass time, min & $90.57 \pm 37.69$ & $98.83 \pm 10.96$ & 0.599 \\
Cross-clamp time, min & $66.26 \pm 31.01$ & $80.16 \pm 11.08$ & 0.285 \\
$\begin{array}{l}\text { Total operation time, } \\
\text { min }\end{array}$ & $275.85 \pm 69.24$ & $282.71 \pm 60.08$ & 0.164 \\
\hline
\end{tabular}

a Abbreviations: R, Remifentanil; P, Paracetamol.

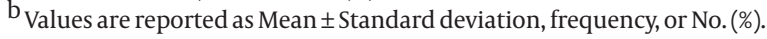

\begin{tabular}{|c|c|c|c|c|c|c|}
\hline & $\mathrm{BP}, \mathrm{mmHg}$ & PValue & HR, beat/min & P Value & RR, breath/min & PValue \\
\hline 2nd hour & & 0.993 & & 0.292 & & $<0.001$ \\
\hline Group R & $117.59 \pm 23$ & & $94.30 \pm 19$ & & $11.8 \pm 1.70$ & \\
\hline Group P & $117.62 \pm 23$ & & $90.38 \pm 18$ & & $15 \pm 12.45$ & \\
\hline 4th hour & & 0.772 & & 0.434 & & $<0.001$ \\
\hline Group R & $117.89 \pm 15$ & & $93 \pm 16.6$ & & $13.18 \pm 5.6$ & \\
\hline Group P & $117.02 \pm 15$ & & $90.44 \pm 17$ & & $17 \pm 12.80$ & \\
\hline 6th hour & & 0.300 & & 0.344 & & $<0.001$ \\
\hline Group R & $119.9 \pm 24$ & & $93.80 \pm 19.13$ & & $13.80 \pm 3.45$ & \\
\hline Group P & $115.8 \pm 13.3$ & & $90.36 \pm 17$ & & $18.80 \pm 13.10$ & \\
\hline 8th hour & & 0.525 & & 0.162 & & 0.001 \\
\hline Group R & $123.17 \pm 17.7$ & & $85 \pm 20.57$ & & $15.6 \pm 4.08$ & \\
\hline Group P & $120 \pm 12.7$ & & $90.4 \pm 17.7$ & & $19.54 \pm 11.76$ & \\
\hline 12th hour & & 0.529 & & 0.748 & & $<0.001$ \\
\hline Group R & $120 \pm 15.6$ & & $87.40 \pm 13.7$ & & $16 \pm 3.67$ & \\
\hline Group P & $118 \pm 13.4$ & & $86.34 \pm 18.8$ & & $20.32 \pm 11.70$ & \\
\hline 18th hour & & 0.515 & & 0.814 & & 0.002 \\
\hline Group R & $121.71 \pm 17.65$ & & $84.86 \pm 13.50$ & & $17.14 \pm 3.46$ & \\
\hline Group P & $120 \pm 12.60$ & & $85.60 \pm 16.50$ & 0.319 & $21 \pm 11.70$ & \\
\hline 24th hour & & 0.079 & & & & 0.027 \\
\hline Group R & $123.52 \pm 18.06$ & & $87 \pm 12$ & & $18 \pm 2.30$ & \\
\hline Group P & $118 \pm 12.2$ & & $84.30 \pm 14.31$ & & $20.9 \pm 12.30$ & \\
\hline
\end{tabular}


Table 3. The Intensity of Intensity of Pain After Coronary Artery Bypass Grafting Surgery, visual Analog Scale Assessment ${ }^{\mathrm{a}, \mathrm{b}}$

\begin{tabular}{lccc}
\hline & $\begin{array}{c}\text { VAS, Group R } \\
(\mathbf{n}=\mathbf{5 0})\end{array}$ & $\begin{array}{c}\text { VAS, Group P } \\
(\mathbf{n}=\mathbf{5 0})\end{array}$ & P Value \\
\hline 2nd hour & $0.68 \pm 1.80$ & $0.60 \pm 1.01$ & 0.425 \\
4th hour & $1 \pm 2$ & $0.34 \pm 0.71$ & 0.085 \\
6th hour & $1.10 \pm 1.91$ & $0.48 \pm 0.76$ & 0.329 \\
8th hour & $1.08 \pm 1.92$ & $0.28 \pm 0.60$ & 0.031 \\
12th hour & $0.86 \pm 1.52$ & $0.34 \pm 0.74$ & 0.133 \\
18th hour & $1.04 \pm 1.80$ & $0.30 \pm 0.78$ & 0.023 \\
24th hour & $0.76 \pm 1.51$ & $0.26 \pm 0.63$ & 0.194 \\
\hline a Abbreviations: $R$, Remifentanil P, Paracetamol, VAS, Visual Analog \\
$\begin{array}{l}\text { Score. } \\
\text { b Values are reported as Mean } \pm \text { Standard deviation. }\end{array}$
\end{tabular}

\section{Discussion}

Adequate analgesia within the first hours of early postoperative period in order to control the pain is very important for a good prognosis of pain syndrome and to prevent its progression to its chronic form (10). Different analgesics are used and different techniques are performed in order to achieve this goal. One of these analgesics is remifentanil. The pharmacodynamic and pharmacologic characteristics of remifentanil make this opioid work as a proper as other analgesic after cardiac surgery. Remifentanil is an ultra-short-acting opioid with more analgesic effect than morphine. It is metabolized by non-specific esterase and its metabolism is not dependent on liver or renal function (3). The other analgesic is paracetamol which is a non-opioid analgesic. The

\begin{tabular}{|c|c|c|c|c|c|c|c|c|}
\hline & PH & P Value & $\mathrm{PaCO}_{2}, \mathrm{mmHg}$ & PValue & $\mathrm{PaO}_{2}, \mathrm{mmHg}$ & P Value & Lactate, $\mathrm{mmol} / \mathrm{L}$ & P Value \\
\hline 2nd hour & & 0.877 & & 0.246 & & 0.007 & & 0.048 \\
\hline Group R & $7.34 \pm 0.08$ & & $32.47 \pm 7.05$ & & $107.76 \pm 33.82$ & & $5.35 \pm 12.31$ & \\
\hline Group P & $7.34 \pm 0.05$ & & $34.32 \pm 6.46$ & & $127.50 \pm 37.98$ & & $4.46 \pm 2.25$ & \\
\hline 4th hour & & 0.115 & & 0.145 & & 0.037 & & 0.005 \\
\hline Group R & $7.35 \pm 0.06$ & & $33.72 \pm 6.04$ & & $109.96 \pm 30.89$ & & $5.20 \pm 13.77$ & \\
\hline Group P & $7.33 \pm 0.06$ & & $34.10 \pm 5.45$ & & $123.10 \pm 31.21$ & & $4.24 \pm 2.25$ & \\
\hline 6th hour & & 0.943 & & 0.940 & & 0.019 & & 0.049 \\
\hline Group R & $7.36 \pm 0.05$ & & $34.72 \pm 6.09$ & & $104.08 \pm 30.32$ & & $9.32 \pm 32.56$ & \\
\hline Group P & $7.36 \pm 0.05$ & & $34.62 \pm 7.16$ & & $118.78 \pm 31.54$ & & $5.83 \pm 14.88$ & \\
\hline 8th hour & & 0.080 & & 0.354 & & 0.003 & & 0.698 \\
\hline Group R & $7.37 \pm 0.04$ & & $33.90 \pm 5.60$ & & $93.72 \pm 26.02$ & & $3.11 \pm 2.23$ & \\
\hline Group P & $7.38 \pm 0.04$ & & $34.98 \pm 5.98$ & & $111.58 \pm 33.21$ & & $2.90 \pm 1.38$ & \\
\hline 12th hour & & 0.235 & & 0.053 & & 0.007 & & 0.981 \\
\hline Group R & $7.40 \pm 0.08$ & & $34 \pm 4.59$ & & $89.28 \pm 26.21$ & & $3.01 \pm 2.69$ & \\
\hline Group P & $7.38 \pm 0.06$ & & $35.96 \pm 5.39$ & & $105.56 \pm 32.89$ & & $2.44 \pm 1.34$ & \\
\hline 18th hour & & 0.730 & & 0.141 & & 0.021 & & 0.047 \\
\hline Group R & $7.40 \pm 0.55$ & & $34.30 \pm 7.14$ & & $79.62 \pm 24.29$ & & $2.54 \pm 1.72$ & \\
\hline Group P & $7.34 \pm 0.42$ & & $36.16 \pm 5.23$ & & $89.76 \pm 25.85$ & & $1.92 \pm 1.31$ & \\
\hline 24th hour & & 0.844 & & 0.433 & & 0.268 & & 0.033 \\
\hline Group R & $7.42 \pm 0.05$ & & $34.44 \pm 6.83$ & & $81.18 \pm 26.33$ & & $2.01 \pm 1.45$ & \\
\hline Group P & $7.42 \pm 0.03$ & & $35.38 \pm 4.95$ & & $87.14 \pm 27.19$ & & $1.53 \pm 1.37$ & \\
\hline
\end{tabular}

\footnotetext{
${ }_{\mathrm{a}}^{\mathrm{a}}$ Abbreviations: $\mathrm{R}$, remifentanil; $\mathrm{P}$, paracetamol; $\mathrm{PaO}_{2}$, partial pressure of arterial oxygen; $\mathrm{PaCO}_{2}$, partial pressure of arterial carbon dioxide.

$\mathrm{b}_{\text {Values are reported as Mean } \pm \text { Standard Deviation. }}$
}

\begin{tabular}{lccc}
\hline Table 5. Patient Complications & \\
\hline a,b & Group R (n= 50) & Group P(n= 50) & P Value \\
\hline Atelectasis & $15(30)$ & $13(26)$ & 0.656 \\
Respiratory Depression & - & - & - \\
Nausea & $14(28)$ & $8(16)$ & 0.148 \\
Vomiting & $4(8)$ & $2(4)$ & 0.400 \\
Itching & - & - & - \\
Myocardial ischemia & $5(10)$ & $5(10)$ & 1.000 \\
Liver dysfunction & - & - & - \\
Renal dysfunction & $14(28)$ & $12(24)$ & 0.647 \\
\hline
\end{tabular}

a Abbreviations: R, Remifentanil; P, Paracetamol.
b Values are reported as No.(\%). 
adverse effects caused by opioids are not reported after paracetamol administration. However, the efficacy of paracetamol used in cardiac surgery is not well-established in the literatures and the results of the studies are conflicting (10). In this study, the intensity of pain was assessed in two groups (group R: remifentanil and group P: paracetamol) by VAS in the first 24-hour period after the surgery. In both group of our study, there were effective pain reduction, which showed adequate pain relief ability in both analgesics. Pettersson et al. compared oral and intravenous administration of paracetamol in reducing the use of opioids after cardiac surgery and they concluded that less opioids were needed in group of patients with intravenous paracetamol administration due to the adequate pain relief effect of intravenous paracetamol (11). Also, the study of Baltali et al. revealed that PCAremifentanil technique provided the effective pain score $<3$ in patients underwent CABG (3). The results of our study also showed that the mean value of VAS was lower in group P comparing to group $\mathrm{R}$ in all seven evaluating times, but only at 8 hours when most of the patients were weaned from mechanical ventilation and 18 hours after the surgery, this difference was significant. The mean value of hemodynamic variables; BP and HR were similar between groups, though the mean value of RR was significantly lower in all seven evaluating times in group $R$ than group $\mathrm{P}$, that is because of remifentanil is reported as a potent respiratory depressant agent in different studies (3). However, in our study, no respiratory depression (RR $<8$ ) was reported. The same results were observed in the study by Gurbet et al. (4) and Krishnan et al. (5) that no episodes of respiratory depression were reported in their study after administration of PCA-remifentanil. The duration of intubation did not statistically differ between groups in our study. This suggests that remifentanil with IVPCA does not prolong the time to intubation after CABG like the results of the study of Gurbet et al. (4). Also IVPCAparacetamol does not have side effects with regard to the duration of intubation.

The results of the $A B G$ analysis revealed that the mean values of post-operative $\mathrm{PH}$ and $\mathrm{PaCo} 2$ were similar between groups. On the other hand, the mean value of $\mathrm{PaO} 2$ was significantly lower in group $\mathrm{R}$ in 2, 4, 6, 8, 12, 18 hours after the surgery. This variable was also reported lower in group R comparing to group P in 24 hours after the operation but with no significant difference. In addition; there were no significant differences in the incidence of adverse effects between groups. In group R, 28\% of the patients suffered from nausea vs. 14\% in group P similar to the study of Baltali et al., that the incidence of nausea in the first 24 hours after the surgery in group of patients who received remifentanil was reported $28.9 \%$ (3) and in the study of Gurbet et al. this complication was reported $20 \%$ in the remifentanil group (4). The incidence of vomiting in the first postsurgical 24 hours in our study was higher in group R (8\%) than group P (4\%) with no significant difference; however the incidence of this variable was reported higher in remifentanil group (14.8\%) in the study of Baltali et al. (3) than our study. The incidence of atelectasis in the first 24 hours after the surgery was observed in $30 \%$ in remifentanil group vs. $26 \%$ in paracetamol group with no significant difference, as in the study of Baltali et al. this complication was reported lower (14\%) in remifentanil group (3). Postoperative myocardial ischemia was observed in $10 \%$ of the patients in each group. This event was reported higher in the study of Checketts et al. (12) which was $19 \%$ in the group of patients who received alfentanil instead of morphine. Postoperative serum $\mathrm{Cr}$ level $>2$ was reported in $24 \%$ of the patients in group $\mathrm{P}$ vs. $28 \%$ in group R with no significant difference. None of the patients suffered from renal failure that resulted into dialysis. Hepatic dysfunction was also not occurred in our study groups. Based on the results of this study, we concluded that both PCA techniques (remifentanil and paracetamol) provided the effective pain score $<3$ with no significant complications. Both PCA remifentanil and paracetamol are safe alternatives for the patients in whom PCA morphine is not suitable after CABG surgery. The results of this study also showed that paracetamol had better pain relief effect and outcomes than remifentanil and it may be administered as a proper analgesic following cardiac surgeries. To become certain of this matter we suggest to conduct a study with more cases and follow up the patients to the time of the ICU discharge.

\section{Acknowledgements}

We would like to acknowledge everyone participated in this study especially anesthesiology department of Shahid Rajaee Heart Hospital.

\section{Authors' Contributions}

All of authors participated in this article.

\section{References}

1. Kim K, Kim WJ, Choi DK, Lee YK, Choi IC, Sim JY. The analgesic efficacy and safety of nefopam in patient-controlled analgesia after cardiac surgery: A randomized, double-blind, prospective study. J Int Med Res. 2014;42(3):684-92.

2. Sattari M, Baghdadchi ME, Kheyri M, Khakzadi H, Ozar Mashayekhi S. Study of patient pain management after heart surgery. Adv Pharm Bull. 2013;3(2):373-7.

3. Baltali S, Turkoz A, Bozdogan N, Demirturk OS, Baltali M, Turkoz $\mathrm{R}$, et al. The efficacy of intravenous patient-controlled remifentanil versus morphine anesthesia after coronary artery surgery. $J$ Cardiothorac Vasc Anesth. 2009;23(2):170-4.

4. Gurbet A, Goren S, Sahin S, Uckunkaya N, Korfali G. Comparison of analgesic effects of morphine, fentanyl, and remifentanil with intravenous patient-controlled analgesia after cardiac surgery. $J$ Cardiothorac Vasc Anesth. 2004;18(6):755-8.

5. Krishnan K, Elliot SC, Berridge JC, Mallick A. Remifentanil patient-controlled analgesia following cardiac surgery. Acta Anaesthesiol Scand. 2005;49(6):876-9.

6. Yarmush J, D'Angelo R, Kirkhart B, O'Leary C, Pitts MC, 2nd, Graf $\mathrm{G}$, et al. A comparison of remifentanil and morphine sulfate for acute postoperative analgesia after total intravenous anesthesia with remifentanil and propofol. Anesthesiology.1997;87(2):235-43.

7. Bowdle TA, Camporesi EM, Maysick L, Hogue CW, Jr, Miguel RV, Pitts M, et al. A multicenter evaluation of remifentanil for early 


\section{Jahangiri Fard A et al.}

postoperative analgesia. Anesth Analg. 1996;83(6):1292-7.

8. Rauf K, Vohra A, Fernandez-Jimenez P, O'Keeffe N, Forrest M. Remifentanil infusion in association with fentanyl-propofol anaesthesia in patients undergoing cardiac surgery: effects on morphine requirement and postoperative analgesia. $\mathrm{Br} \mathrm{J} \mathrm{An-}$ aesth. 2005;95(5):611-5.

9. Hinz B, Cheremina O, Brune K. Acetaminophen (paracetamol) is a selective cyclooxygenase-2 inhibitor in man. FASEB $J$. 2008;22(2):383-90.

10. Eremenko AA, Kuslieva EV. [Analgesic and opioid-sparing effects of intravenous paracetamol in the early period after aortocoronary bypass surgery]. Anesteziol Reanimatol. 2008(5):11-4.

11. Pettersson PH, Jakobsson J, Owall A. Intravenous acetaminophen reduced the use of opioids compared with oral administration after coronary artery bypass grafting. J Cardiothorac Vasc Anesth. 2005;19(3):306-9.

12. Checketts MR, Gilhooly CJ, Kenny GN. Patient-maintained an algesia with target-controlled alfentanil infusion after cardiac surgery: a comparison with morphine PCA. Br J Anaesth. 1998;80(6):748-51. 